
\title{
R Reserach S Suare \\ Whole-genome identification of chemosensory genes in two sister blister beetles provides insights into chemosensory evolution
}

Yuanming Wu ( $\nabla$ wuyuanming@gmc.edu.cn )

Guizhou Medical University

Yang-Yang Liu

Guizhou University

Xiang-Sheng Chen

Guizhou University

Research article

Keywords: blister beetles (Spanish fly), chemosensory, gustatory receptor, odorant receptor, ionotropic receptors

Posted Date: August 27th, 2019

DOI: https://doi.org/10.21203/rs.2.13579/v1

License: (c) (i) This work is licensed under a Creative Commons Attribution 4.0 International License. Read Full License 


\section{Abstract}

Background: Commonly known as blister beetles or Spanish fly, there are more than 1,500 species in the Meloidae family (Coleoptera:Tenebrionoidea) that produce the potent defensive blistering agent cantharidin, which has recently been exploited for cancer therapy. Hycleus cichorii and Hycleus phaleratus are exploited as traditional Chinese medicine over 2,000 years due to their ability to biosynthesize cantharidin. These blister beetles share highly similar environments and ecological niches. To understand the role of the chemosensory system in speciation and evolution in the beetles, we identified the chemosensory gene families in whole genome of both blister beetle comprehensively. Results: We identified 29 OBPs, 10 CSPs, 116 ORs, 80 GRs, and 35 IRs in H. phaleratus and 30 OBPs, 10 CSPs, 191 ORs, 92 GRs and 42 IRs in H. cichorii. The 7 groups of beetles' ORs were recovered, but a new pattern of ORs in Coleoptera were surfaced, due to lack of whole OR repertoire for comparison to T. castaneum before. The OR number is varied, identified in $\mathrm{H}$. cichorii $64 \%$ more than that in $\mathrm{H}$. phaleratus (191 VS 116), which maybe an evolution event between these blister beetle's speciation. A major Hycleusspecific expansion clade of bitter GRs is evident based on our phylogenetic tree, in which, clustered more than $50 \%$ Hycleus-bitter GRs. All the ten clade of beetles' antennal IR identified in both Hycleus beetles. Interestingly, IR75 and IR41a were obviously expensed compare to other insects. Conclusions: The GR and IR expansion events may promote Hycleus genus evolution. Our data will provide a basis for future species protection and speciation focusing on the blister beetle.

\section{Background}

To adapt to varied environments and ecological niches, insects always rely upon their sensitive olfactory system to distinguish crucial chemical signals, such as pheromones and plant volatiles. Because olfaction is an important element of host and mate selection, the identification of changes in chemosensory genes between diverging populations or closely related species can provide valuable insights into the role of chemosensory adaptation in different environments and host speciation [1]. At the species level and above, gene amplification and amino acid mutation events may play essential roles during adaptive evolution. For example, some gustatory receptor (GR) and odorant receptor (OR) genes underwent rapid evolution during host specialization in Drosophila [2], and certain chemoreceptor genes with genetic divergence might play key roles in local adaptation and reproductive isolation in the pea aphid Acyrthosiphon pisum [3]. Using developing expression analysis and targeted mutagenesis experiments, Matsuo et al. [4] identified two genes encoding odorant binding proteins (OBPs) that were responsible for behavioral differences between Drosophila sechellia and its sibling species in their responses to taste perception and host plant preferences. Moreover, the evolution of differential gene expression may act as a key mechanism of chemosensory divergence. For instance, a comparison of chemosensory gene expression profiles of $D$. sechellia and two sibling species showed significant changes in gene expression that might be related to host specialization [5].

In insects, at least five gene families are involved in the detection of chemical signals. These families include three receptor gene families [ORs, ionotropic receptors (IRs) and GRs], and two binding protein 
gene families [OBPs and chemosensory proteins (CSPs)], [6-10]. The receptor gene families are usually expressed in the olfactory sensory neurons and are involved in the detection of volatile chemicals (ORs) [11-14], contact chemicals or carbon dioxide (GRs) [15,16], and nitrogen-containing compounds, acids, and aromatics (IRs) [17]. In contrast, the binding protein gene families are highly abundant in the sensillum lymph and usually function as binding of molecules that interact with receptors $[18,19]$. Together, these genes allow insects to seek and select hosts and mates and thus affect the adaptability changes in closely related insect species. Although several of studies have compared differences in chemosensory genes between sister species [20-27], comparatively few cases have aimed to understand the mechanisms that have shaped divergence or chemosensory speciation. Chemical cues with diverse ecological functions can be detected by focusing on the chemosensory genes expressed in the insects $[28,29]$. Moreover, key genetic factors can impact insect behavior and host use.

Commonly known as blister beetles or Spanish fly, there are more than 2,500 species in the Meloidae family, with more than 1,500 of these beetle species known to produce cantharidin. Cantharidin $\left(\mathrm{C}_{10} \mathrm{H}_{12} \mathrm{O}_{4}\right)$ is widely used in anti-insect and bacterial products in applications in agriculture and medicine worldwide [30-32]. Recently, cantharidin and its derivatives have been used to treat several cancers, including stomach, liver, lung and esophageal cancers [33-35]. The dry body of this beetle has been a traditional medicine in China for the past 2000 years. However, only Hycleus cichorii Linnaeus and Hycleus phaleratus Pallas are broadly used and listed in the Pharmacopoeia of the People's Republic of China [36].

These two beetles have largely overlapping sympatric ranges in China and a similar emergence phenology and appearance, except that $H$. phaleratus has a bigger body size. The beetles are mainly distributed in southwest China and feed on the eggs of Locustidae during the larvae period and on legumes and cucurbitaceous crops in adulthood. Therefore, understanding the changes that underlie the speciation between these two sister species and comparing the chemosensory proteins of extremely importance for the insects' survival and reproduction are interesting strategy. On other hand, in Coleoptera groups, the chemosensory genes have rarely been identified in whole genome except the flour beetle $T$. castaneum [37,38], lack of enough data for comprehensive analysis of gene evolution. Here, we identified the members of five gene families involved in chemosensory perception in Hycleus genomes and analysed its evolution in beetles.

\section{Methods}

\section{Identification of chemosensory genes}

The $H$. cichorii and $H$. phaleratus genome sequences were obtained from the GigaScience Database [39]. We identified the OBP, CSP, OR, GR, and IR genes using the following steps. First, we constructed a protein reference database of each family that included two parts: Dendroctonus ponderosae, Tribolium castaneum, Pyrrhalta aenescens and Pyrrhalta maculicollis, Chrysomela lapponica from GenBank and Acyrthosiphon pisum, Drosophila melanogaster, Bombyx mori, Apis mellifera, Dendroctonus ponderosae, 
and Tribolium castaneum from InsectBase. The second, a tBLASTn (Version 2.2.25) alignment was used to search the chemosensory genes onto both genome with an e-value cut-off of $1 e-5$. The third, we first linked the alignment hits into candidate gene loci exon by exon with house Perl script. We then extracted genomic sequences of candidate loci, together with $1 \mathrm{~kb}$ flanking sequences, using Gene-Wise [40] to determine gene models. Next, the conflict region was manually compared with other gene of the same family. At last, we checked the functional annotations of the public data and removed candidate genes that had inconsistent functions. Finally, repeat the whole processes used the identified putative proteins to search again, incase missing the new genes.

\section{Phylogenetic tree and selection analysis}

To analyze the characteristics of the Hycleus chemosensory genes and their relationships to other insects, $M L$ trees for each family were constructed using the amino acid sequences derived from the putative CDSs and the published sequences of other species, including T. castaneum, D. ponderosae, C. lapponica and $D$. melanogaster. The published sequences were retrieved from NCBI and InsectBase (S1 Dataset). First, a multiple sequence alignment was generated through MAFFT (Version 7. 407) [41] using the chemosensory gene amino acid sequences of 6 species. Then, RAxML (v8.2.12) [42] was used to construct chemosensory phylogenetic trees with best models (LG for CSPs and OBPs, WAG for IPs, JTT for ORs and GRs), and node support was assessed using a bootstrap procedure based on 2,00 replicates. We viewed the phylogenetic tree with EvolView (http://evolgenius.info/evolview).

According to the phylogenetic tree, we selected the orthologous genes between these two Hycleus species to process in the selection analysis. The paired orthologs of the protein sequences were aligned using MAFFT (version 7.407) [41]. Then, the alignment information for the protein sequence was used as a guide for the nucleic acid sequence alignment. Finally, analyses of nonsynonymous (KA) and synonymous (KS) substitutions were performed by Kaks_Calculator (v2.0) [43] using the YN method.

\section{RNA-seq for gene profiling}

The RNA-seq data of $H$. phaleratus (whole body and antennal) are from the NCBI SRA database (BioProject accession number PRJNA381455). We mapped the reads onto the integrated gene set using the Bowtie2 (version v2.2.5) [44] software and then calculated the transcript expression levels (fragments per kilobase per million mapped fragments [FPKM]) with the RSEM (version v1.2.12) software [45]. The following parameters were used in Bowtie: (1) q, phred64; (2) sensitive, dpad 0; (3) gbar, 99999999; (4) $\mathrm{mp}, 1,1$; (5) np 1; (6) score-min L,0, 0.1 -I 1 -X 1000; (7) no-mixed; and (8) no-discordant -p 1 -k 200.

\section{Results}

The annotated candidate chemosensory genes were identified by Blastp and functional verification. In total, we identified 29 OBPs, 10 CSPs, 116 ORs, 80 GRs, and 35 IRs in H. phaleratus and 30 OBPs, 10 
CSPs, 191 ORs, 92 GRs and 42 IRs in H. cichorii (Table 1 and Additional file 1). Moreover, 23 out of 29 OBPs, 23 out of 29 CSPs, 47 out of 116 ORs, 30 out of 80 GRs, and 26 out of 48 iGluRs/IRs have been expressed (FPKM $\geq 1$ ) in whole body or antennal tissue of $H$. phaleratus (Additional files 2). All the CDSs and proteins identified in this study was listed in Additional file 3 and Additional file 4.

\section{OBPs.}

We identified 29 and 30 OBPs in the two blister beetles, respectively; and 48 out of 59 OBPs with nearly full-length CDSs. We identified 28 classic, 4 plus-C and 27 minus-C OBPs based on their sequence properties $[18,46,47]$. The maximum likelihood (ML) phylogenetic tree suggested 28 pairs of orthologous OBPs between the two beetles (Fig S1). Analysis of selection performed on the orthologs between the two blister beetles suggested that 13 pairs ortholog ( $d N / d S \leq 0.3436$, Fisher's test $P \leq 0.05$ ) was under purifying selection (Additional files 1).

\section{Chemosensory proteins.}

We identified ten CSPs in $H$. phaleratus and $H$. cichorii, respectively; all the CSPs represented full-length proteins including the conserved C-pattern, $\mathrm{C}_{1} \mathrm{X}_{6} \mathrm{C} 2 \mathrm{X}_{18} \mathrm{C} 3 \mathrm{X}_{2} \mathrm{C} 4$ [47]. Nine pairs of chemosensory protein orthologs were shared by the two blister beetles (Fig S2). The selection pressure analysis showed that purifying selection acted on six CSP orthologs (Additional files 1).

\section{Odorant receptors.}

The coleopteran OR subfamilies were classified by Engsontia et al. [37] and Andersson et al. [20]. Based on previous studies, seven groups were recovered in this study (Fig. 1). Group 1 and 6 contained ORs were mainly Tribolium castaneum-specific except for a few ORs. Group 2 contained a mixture of ORs from all five species. Group 3 and 5 only contain ORs mainly from Tribolium castaneum and Hycleus genus. Groups 4 were Tribolium castaneum-specific clade. Otherwise, group 7 was a specific expansion in bark beetles (DponOR) and blister beetles, and this clade exception ORs of Tribolium castaneum (Fig. 1). Specifically, HcicORco and HphaORco were identified, and ORco homolog group was clustered into group 7 (Fig. 1).

Notably, we identified 116 and 191 ORs in $H$. phaleratus and $H$. cichorii genome, and up to 64\% ORs identified in $H$. cichorii more than that in its sister species. This expensed ORs may acts a key functional along to its species speciation.

We identified 86 pairs of orthologs between the two beetles based on the ML tree, in which, 49 pairs of orthologs were under purifying selection (Additional files 1).

\section{Gustatory receptors.}


We identified 80 and 92 GRs in H. phaleratus and H. cichorii, respectively. And 63 pairs of GRs ortholog were shared by the two blister beetles ( $\mathrm{Fig} 2$ ). Three pairs of $\mathrm{CO}_{2}$ receptors were identified, and one by one orthologs presents among Hycleus genus and T. castaneum (Fig. 2). Eight and seven sugar receptors, which was in DmelGR64a-f cluster, were detected in this study (Fig. 2). Also, a pair of homologs (HcicGR56 and HphaGR56) were homolog to DmGR43a, which functions as a fructose receptor. Notably, a huge Hycleus-specific expansion clade is evident in our phylogenetic tree (Fig. 2). More than $1 / 2$ identified Hycleus bitter GR were classified in this clade, that may act important functions in Hycleus genus speciation. Moreover, three species (or genus) expansion clade, Hycleus genus, T.castaneum, and D. melanogaster, were identified (Fig. 2). A total of 40 pairs of orthologs between blister beetles were under purifying selection (Additional files 1).

\section{lonotropic receptors.}

IRs are more closely related to ionotropic glutamate receptors (iGluRs) $[38,48]$. A total of 35 and 42 IRs and 13 and 13 iGluRs were identified in $H$. phaleratus and $H$. cichorii, respectively (Table 1 ). The DmelIR25a and DmelIR8a was considered the coreceptor IRs, and their orthologs of both species of Hycleus were identified in this study (Fig. 3). It is very conserved from beetle to fruit fly, a 1:1 orthologs appeared in our $\mathrm{ML}$ tree. This cluster are in the clade of iGluRs, corresponds to previous report [48,49]. All the ten of beetle's antenna IRs, including IR75, IR64a, IR40a, IR21a, IR68a, IR60a, IR41a, IR76b and IR93a, were identified (Fig 3 and Table 2). Eight of ten clades of these only detected one copy in both blister beetles, and five of that were 1:1 ortholog in all the six insects (Fig 3 and Table 2). Notably, a total of 11 and 15 copies of IR41a were annotated in $H$. phaleratus and $H$. cichorii, respectively. However, that corresponding IR in other species no more than 2 copies (Fig 3 and Table 2). It was expensed obviously in blister beetles compare to other insects. IR41a is not a unique instance. Also, the IR75 were expensed in both blister beetles relatively, 10 and 16 copies in these two beetles but up to 4 copies in other insects (Fig 3 and Table 2).

A total of 35 pairs of orthologous iGluRs/IRs were found between both species, and 30 pairs of orthologs were under purifying selection (Additional files 1).

\section{Discussion}

Chemosensory traits are key components of speciation in many insects, and changes in these traits can influence insect behavior and subsequently mediate survivability. Although evaluating changes in chemosensory gene is a necessary beginning for understanding how chemosensory traits contribute to adaptability, surprisingly few studies have compared how sister taxa differ in this field. Moreover, chemosensory gene identification within Coleoptera groups has rarely been reported. These genes have been characterized in whole genome level only the flour beetle T. castaneum [37]. Several species have been identified partly by RNA-seq, such as two bark beetles (I. typographus and $D$. ponderosae) [20], two leaf beetles ( $P$. aenescens and $P$. maculicollis) [27], scarab beetle Hylamorpha elegans [50], and a leaf 
beetle $C$. lapponica [49]. In the present study, we identified the chemosensory gene families at whole genome level in a pair of blister beetles (Meloidae: Hycleus) in the Meloidae and provided the evolution analysis of chemosensory genes within Coleoptera groups.

\section{Non-receptor gene families}

The OBP and CSP families mediate the first critical step of chemosensation in insects. Thus, these genes have received considerable attention $[27,47,50-55]$. When chemical signals enter the sensillum lymph, OBPs/CSPs bind it and involve in peripheral olfactory processing [27]. Our analysis of these gene families obtained 29 and 30 OBPs and 10 and 10 CSPs in H. phaleratus and H. cichorii, respectively. These totals are comparable to the totals observed in previous reports, such as in the $D$. ponderosae transcriptome (31 OBPs and 11 CSPs) [20], the Pyrrhalta genus transcriptome (31 and 36 OBPs and 9 and 10 CSPs) [27] and the leaf beetle $C$. lapponica (32 OBPs and 12 CSPs)[49]. An expansion of the minus-C OBPs was found in this Hycleus genus (27 of minus-C OBPs) comparison to D. melanogaster, the similar pattern reported in Tribolium and bark beetles [20], two beetles in the Pyrrhalta genus [27] and the leaf beetle $C$. lapponica [49]. The expanded Minus-C OBPs may one specific evolution event in Coleoptera chemosensory, and it suggests that these genes might play an important role in chemosensation.

\section{Receptor gene families}

The OR family, which was studied mostly in these three receptors. Based on previous studies [20,37], seven groups of beetle ORs were recovered in this study. Notably, some new patterns have been found in this study (Fig. 1). Such as, the group 7 was a specific expansion only in bark beetles (DponOR and ItypOR) was reported before [20]; this clade was also expansion in blister beetles was be found in our result. The group 3 and 5 almost the flour beetle specific ORs was reported before [20,25, 27], but we found lots of Hycleus ORs also in these clades. This may due to the most reports were identified ORs via RNA-seq except T. castaneum (341) [37]. These species only tens of ORs could been found in RNA-seq, such as the leaf beetles $P$. maculicollis (22) and P. aenescens26) [27], the bark beetles I. typographus (43) and $D$. ponderosae (49) [20], the long horned beetle Megacyllene caryae (57) [25], and the leaf beetle $C$. lapponica (42)[49]. This indicted more beetle's chemosensory family should be annotated comprehensively via whole genome that is better for comprehensive study its evolution in insects.

Moreover, the number of ORs in each blister beetles was obvious differ, ORs identified in $\mathrm{H}$. cichorii $64 \%$ more than that in H. phaleratus (191 VS 116). However, the number of others only slightly vary (Table 1). These data hint ORs specific expansion in $H$. cichorii along both specie differentiation, which occurred around 23 million year ago [56]. ORs is one of the key bridges between animals and its surrounding, and act functions with food, predators and potential mates. These two beetles have largely overlapping sympatric ranges in west south of China. To our field survey experience, in the past years, the $H$. phaleratus population has declined in the field due to destruction of the living environment by human activity and human catching. In contrast, the $H$. cichorii population has not declined obviously as the 
same situation. These ORs maybe acts the keys roles for a stronger adaption ability than H. phaleratus. These ORs needs verified in the future.

For GRs, always divides into three groups by its recognizing materials, $\mathrm{CO}_{2}$, sugar and better receptors. we identified three pairs of $\mathrm{CO}_{2}$ receptors, and nine and eight sugar receptors in two blister beetles (Fig 2). These two groups always more conservative in insects. Notably, a Hycleus-specific expansion clade was found in our phylogenetic tree, and more than 1/2 identified Hycleus-bitter GR were in this clade. That may act important functions in Hycleus genus. On the other hand, there are lack whole GRs identify from genome of other species for comparative analysis. Most published GR family by RNA-seq, only few GRs was been detected due to the GRs expressed very low. such as in P. aenescens (16), P. maculicollis (10) [27], I. typographus (2) and D. ponderosae (6) [20], the leaf beetle $C$. lapponica (8)[49].

IRs is also membrane proteins, are more closely related to ionotropic glutamate receptors (iGluRs) $[38,48]$. We identified 35 and 42 IRs in $H$. phaleratus and $H$. cichorii. IRs could be divided into two groups: 'antennal IRs' was always conserved among species, which likely define the olfactory receptor family of insects, and the 'divergent IRs' was more divergent, which are expressed in peripheral and internal gustatory neurons, implicating this family in taste and food assessment [38]. All the ten clades of beetle antennal IRs were identified in both blister beetles, and five of these sub-family were conserved with 1:1 ortholog in all the six insects (Fig. 3 and Table 2). However, the IR41a (11 and 15 in blister beetles VS 1-2 in others) and IR75 (10 and 16 in blister beetles VS 3-4 in others) were evidently expensed compare to others (Fig 3 and Table 2). Moreover, there have 5 out 11 copies of IR41a and 7 out of 10 copies of IR75 were detected expression (FPKM $\geq 1$ ). This data hints that IR41a and IR75 would be specific expansion in the Hycleus genus, that could introduce new characteristics for the Hycleus genus. We first time identified IR60a (HphalR60a1 and HphalR60a2) in the beetles based on our phylogenetic tree, it missed in $T$. castaneum and other beetles. Additionally, HphalR60a1 has expressed (FPKM: 2.53- 8.81) in antennal tissues. This gene should be verified in the future study.

\section{Candidate chemosensory genes for speciation}

Divergent chemosensory genes linked to differences in the sensory tuning of sister species represent a response to divergent selection of chemosensory traits [1]; thus, these genes are candidates for chemicalmediated speciation, which directly or indirectly reflects the ability to adapt to the environment. For instance, a comparison of the gene amplification of chemosensory gene families between the sister beetle species suggested some candidate genes that might have been involved in this ability. In present study, an evident expansion of ORs identified in H. cichorii compare to that in H. phaleratus (191 VS 116). These data hint ORs specific expansion in H. cichorii may promoted both specie differentiation. Which specific ORs and how acts the keys role should be study in the next. On the other hands, we identified 221 pairs of gene between both blister beetles, of which 138 pairs were undergone purifying selection and no gene under positive selection (Additional files 1). This data may hint no positive clue of gene mutation 
linking to the speciation, the other ways should be attended, such as gene expression change. At least, expression changes between orthologs should be attended in the future study.

\section{Conclusions}

In the present study, we firstly performed a comprehensive whole genome identification of a pair of blister beetles (Meloidae: Hycleus) with a focus on chemosensory gene families. A New pattern of ORs in Coleoptera were surfaced, due to lack of whole OR repertoire for comparison to T. castaneum before. Several fascinating evolution events in blister beetles was disclosed, such as a clade of Hycleus-specific expansion bitter-GR and IR75 and IR41a evident expansion in Hycleus, which will help to unfold this genus evolution.

\section{Abbreviations}

GR: gustatory receptor

OR: odorant receptor

IR: ionotropic receptor

iGluR: ionotropic glutamate receptor

CSP: chemosensory protein

OBP: odorant binding protein

ML: Maximum likelihood

CDS: coding region sequence

FPKM: fragments per kilobase per million mapped fragments

\section{Declarations}

\section{Funding}

The study was supported by The National Natural Science Foundation of China (No. 81460576). The study was supported by The Program of Science and Technology Innovation Talents Team, Guizhou Province (No. 20144001). The study was supported by The Program of Excellent Innovation Talents, Guizhou Province (No. 20154021). The study was supported by The International Cooperation Base for Insect Evolutionary Biology and Pest Control (No. 20165802). The funding bodies played no role in the design of the study and collection, analysis, and interpretation of data or in writing the manuscript. 


\section{Availability of data and materials}

All the CDSs and proteins identified in the study was submitted in NCBI GenBank (accession numbers MF385073-MF385135 and MF385136-MF385202.).

\section{Ethics approval and consent to participate}

Not applicable.

\section{Consent for publication}

Not applicable.

\section{Competing interests}

The authors declare that they have no competing interests.

\section{Authors' contributions}

WY and CX design the projects. WY and NY collected the insects and extract the total RNA sample. WY preformed the data analysis and made the figures and tables. WY and CX wrote the manuscript. All authors read and approved the final manuscript.

\section{References}

1. 1.Smadja C, Butlin RK. On the scent of speciation: the chemosensory system and its role in premating isolation. Heredity. 2009;102: 77-97.

2. 2.McBride CS. Rapid evolution of smell and taste receptor genes during host specialization in Drosophila sechellia. Proc Natl Acad Sci U S A. 2007;104: 4996-5001.

3. 3.Smadja CM, Canback B, Vitalis R, Gautier M, Ferrari J, Zhou JJ, et al. Large-scale candidate gene scan reveals the role of chemoreceptor genes in host plant specialization and speciation in the pea aphid. Evolution. 2012;66: 2723-2738.

4. 4.Matsuo T, Sugaya S, Yasukawa J, Aigaki T, Fuyama Y. Odorant-binding proteins OBP57d and OBP57e affect taste perception and host-plant preference in Drosophila sechellia. PLoS Biol. 2007;5: e118.

5. 5.Shiao MS, Chang JM, Fan WL, Lu MY, Notredame C, Fang S, et al. Expression divergence of chemosensory genes between Drosophila sechellia and its sibling species and its implications for host shift. Genome Biol Evol. 2015;7: 2843-2858. 
6. 6.Kaupp UB. Olfactory signalling in vertebrates and insects: differences and commonalities. Nat Rev Neurosci. 2010;11: 188-200.

7. 7.Robertson HM, Kent LB. Evolution of the gene lineage encoding the carbon dioxide receptor in insects. J Insect Sci. 2009;9: 19.

8. 8.Benton R, Vannice KS, Gomez-Diaz C, Vosshall LB. Variant ionotropic glutamate receptors as chemosensory receptors in Drosophila. Cell. 2009;136: 149-162.

9. 9.Touhara K, Vosshall LB. Sensing odorants and pheromones with chemosensory receptors. Annu Rev Physiol. 2009;71: 307-332.

10. 10.Vogt RG, Grosse-Wilde E, Zhou JJ. The lepidoptera odorant binding protein gene family: gene gain and loss within the GOBP/PBP complex of moths and butterflies. Insect Biochem Mol Biol. 2015;62: 142-153.

11. 11.Carey AF, Wang G, Su C-Y, Zwiebel LJ, Carlson JR. Odorant reception in the malaria mosquito Anopheles gambiae. Nature. 2010;464: 66-71.

12. 12. Hallem EA, Carlson JR. Coding of odors by a receptor repertoire. Cell. 2006;125: 143-160.

13. 13.Sakurai T, Nakagawa $T$, Mitsuno $H$, Mori $H$, Endo $Y$, Tanoue $S$, et al. Identification and functional characterization of a sex pheromone receptor in the silkmoth Bombyx mori. Proc Natl Acad Sci U S A. 2004;101: 16653-16658.

14. 14.Stensmyr MC, Dweck HK, Farhan A, Ibba I, Strutz A, Mukunda L, et al. A conserved dedicated olfactory circuit for detecting harmful microbes in Drosophila. Cell. 2012;151: 1345-1357.

15. 15.Kwon JY, Dahanukar A, Weiss LA, Carlson JR. The molecular basis of CO2 reception in Drosophila. Proc Natl Acad Sci U S A. 2007;104: 3574-3578.

16. 16.Vosshall LB, Stocker RF. Molecular architecture of smell and taste in Drosophila. Annu Rev Neurosci. 2007;30: 505-533.

17. 17.Abuin L, Bargeton B, Ulbrich MH, Isacoff EY, Kellenberger S, Benton R. Functional architecture of olfactory ionotropic glutamate receptors. Neuron. 2011;69: 44-60.

18. 18.Sanchez-Gracia A, Vieira FG, Rozas J. Molecular evolution of the major chemosensory gene families in insects. Heredity. 2009;103: 208-216.

19. 19.Zhou JJ. Odorant-binding proteins in insects. Vitam Horm. 2010;83: 241-272.

20. 20.Andersson MN, Grosse-Wilde E, Keeling Cl, Bengtsson JM, Yuen MM, Li M, et al. Antennal transcriptome analysis of the chemosensory gene families in the tree killing bark beetles, Ips typographus and Dendroctonus ponderosae (Coleoptera: Curculionidae: Scolytinae). BMC Genomics. 2013;14: 198.

21. 21.Bengtsson JM, Trona F, Montagne N, Anfora G, Ignell R, Witzgall P, et al. Putative chemosensory receptors of the codling moth, Cydia pomonella, identified by antennal transcriptome analysis. PLoS One. 2012;7: e31620.

22. 22.Grosse-Wilde E, Kuebler LS, Bucks S, Vogel H, Wicher D, Hansson BS. Antennal transcriptome of Manduca sexta. Proc Natl Acad Sci U S A. 2011;108: 7449-7454. 
23. 23.Gu S-H, Zhou J-J, Gao S, Wang D-H, Li X-C, Guo Y-Y, et al. Identification and comparative expression analysis of odorant binding protein genes in the tobacco cutworm Spodoptera litura. Sci Rep. 2015;5: 13800.

24. 24.Legeai F, Malpel S, Montagne N, Monsempes C, Cousserans F, Merlin C, et al. An expressed sequence tag collection from the male antennae of the Noctuid moth Spodoptera littoralis: a resource for olfactory and pheromone detection research. BMC Genomics. 2011;12: 86.

25. 25.Mitchell RF, Hughes DT, Luetje CW, Millar JG, Soriano-Agaton F, Hanks LM, et al. Sequencing and characterizing odorant receptors of the cerambycid beetle Megacyllene caryae. Insect Biochem Mol Biol. 2012;42: 499-505.

26. 26.Zhang S, Zhang Z, Wang H, Kong X. Antennal transcriptome analysis and comparison of olfactory genes in two sympatric defoliators, Dendrolimus houi and Dendrolimus kikuchii (Lepidoptera: Lasiocampidae). Insect Biochem Mol Biol. 2014;52: 69-81.

27. 27.Zhang B, Zhang W, Nie RE, Li WZ, Segraves KA, Yang XK, et al. Comparative transcriptome analysis of chemosensory genes in two sister leaf beetles provides insights into chemosensory speciation. Insect Biochem Mol Biol. 2016;79: 108-118.

28. 28.Dobritsa AA, van der Goes van Naters W, Warr CG, Steinbrecht RA, Carlson JR. Integrating the molecular and cellular basis of odor coding in the Drosophila antenna. Neuron. 2003;37: 827-841.

29. 29.Leal WS. Odorant reception in insects: roles of receptors, binding proteins, and degrading enzymes. Annu Rev Entomol. 2013;58: 373-391.

30. 30.Day RM, Harbord M, Forbes A, Segal AW. Cantharidin blisters: a technique for investigating leukocyte trafficking and cytokine production at sites of inflammation in humans. J Immunol Methods. 2001;257: 213-220.

31. 31.Carrel JE, Eisner T. Cantharidin: potent feeding deterrent to insects. Science. 1974;183: 755-757.

32. Silverberg NB, Sidbury R, Mancini AJ. Childhood molluscum contagiosum: experience with cantharidin therapy in 300 patients. J Am Acad Dermatol. 2000;43: 503-507.

33. 33.Wang CC, Wu CH, Hsieh KJ, Yen KY, Yang LL. Cytotoxic effects of cantharidin on the growth of normal and carcinoma cells. Toxicology. 2000;147: 77-87.

34. 34.Yang H, Guo W, Xu B, Li M, Cui J. Anticancer activity and mechanisms of norcantharidin-Nd3II on hepatoma. Anticancer Drugs. 2007;18: 1133-1137.

35. 35.Liu D, Chen Z. The effects of cantharidin and cantharidin derivates on tumour cells. Anticancer Agents Med Chem. 2009;9: 392-396.

36. 36.Editorial Board of Pharmacopoeia of the People's Republic of China. Pharmacopoeia of the People's Republic of China. Beijing: Chemical Industry Press; 2005.

37. 37.Engsontia P, Sanderson AP, Cobb M, Walden KK, Robertson HM, Brown S. The red flour beetle's large nose: an expanded odorant receptor gene family in Tribolium castaneum. Insect Biochem Mol Biol. 2008;38: 387-397. 
38. 38.Croset V, Rytz R, Cummins SF, Budd A, Brawand D, Kaessmann H, et al. Ancient protostome origin of chemosensory ionotropic glutamate receptors and the evolution of insect taste and olfaction. PLoS Genet. 2010;6: e1001064.

39. 39 Wu Y, Li J, Chen X. Draft genomes of two blister beetles Hycleus cichorii and Hycleus phaleratus. GigaScience Database 2018. http://dx.doi.org/10.5524/100405.

40. Birney E, Clamp M, Durbin R. GeneWise and Genomewise. Genome Res. 2004;14:88-95.

41. Katoh K, Standley DM. MAFFT multiple sequence alignment software version 7: improvements in performance and usability. Mol Biol Evol. 2013;30:772-80.

42. Guindon S, Dufayard JF, Lefort V et al. New algorithms and methods to estimate maximumlikelihood phylogenies: assessing the performance of PhyML 3.0. Syst Biol 2010;59:307-21.

43. Wang D., Zhang Y., Zhang Z., Zhu J., Yu J.. KaKs_Calculator 2.0: a toolkit incorporating gammaseries methods and sliding window strategies.Genomics Proteomics Bioinformatics. 2010 Mar;8(1):77-80.

44. Langmead B, Salzberg SL. 2012. Fast gapped-read alignment with Bowtie 2. Nat Methods, 9: 357359.

45. Li B, Dewey CN. 2011. RSEM: accurate transcript quantification from RNA-Seq data with or without a reference genome. BMC Bioinformatics, 12: 323.

46. 46.Zhou JJ, Huang W, Zhang GA, Pickett JA, Field LM. “Plus-C” odorant-binding protein genes in two Drosophila species and the malaria mosquito Anopheles gambiae. Gene. 2004;327: 117-129.

47. Xu, Y. L., He, P., Zhang, L., Fang, S. Q., Dong, S. L., Zhang, Y. J., \& Li, F. Large-scale identification of odorant-binding proteins and chemosensory proteins from expressed sequence tags in insects. BMC Genomics. 2009:10, 632. https://doi.org/10.1186/1471-2164-10-632

48. Rytz, R., Croset, V., \& Benton, R. (2013). Ionotropic receptors (IRs): Chemosensory ionotropic glutamate receptors in Drosophila and beyond. Insect Biochemistry and Molecular Biology, 43, 888897. https://doi.org/10.1016/j.ibmb.2013.02.007

49. Wang D, Pentzold S, Kunert M, et al. A subset of chemosensory genes differs between two populations of a specialized leaf beetle after host plant shift. Ecol Evol. 2018:8:8055-8075. https://doi.org/10.1002/ece3.4246

50. 50.Venthur H, Zhou J-J, Mutis A, Ceballos R, Mella-Herrera R, Larama G, et al. $\beta$-lonone as putative semiochemical suggested by ligand binding on an odorant-binding protein of Hylamorpha elegans and electroantennographic recordings. Entomol Sci. 2016;19: 188-200.

51. 51.Danty E, Arnold G, Huet JC, Huet D, Masson C, Pernollet JC. Separation, characterization and sexual heterogeneity of multiple putative odorant-binding proteins in the honeybee Apis mellifera $\mathrm{L}$. (Hymenoptera: Apidea). Chem Senses. 1998;23: 83-91.

52. 52.Gong DP, Zhang HJ, Zhao P, Xia QY, Xiang ZH. The odorant binding protein gene family from the genome of silkworm, Bombyx mori. BMC Genomics. 2009;10: 332. 
53. 53.Graham LA, Davies PL. The odorant-binding proteins of Drosophila melanogaster: annotation and characterization of a divergent gene family. Gene. 2002;292: 43-55.

54. 54.Jin X, Brandazza A, Navarrini A, Ban L, Zhang S, Steinbrecht RA, et al. Expression and immunolocalisation of odorant-binding and chemosensory proteins in locusts. Cell Mol Life Sci. 2005;62: 1156-1166.

55. 55.Zhou JJ, Robertson G, He X, Dufour S, Hooper AM, Pickett JA, et al. Characterisation of Bombyx mori odorant-binding proteins reveals that a general odorant-binding protein discriminates between sex pheromone components. J Mol Biol. 2009;389: 529-545.

56. Wu Y, Li J, Chen X. Draft genomes of two blister beetles Hycleus cichorii and Hycleus phaleratus. Gigascience. 2018:7: 1-7. doi: 10.1093/gigascience/giy006.

\section{Tables}

Table 1. The number of chemosensory genes in the genome of the Two Sister Blister Beetles $\mathrm{H}$. phaleratus and $\mathrm{H}$. cichorii. The number in bracket represents expressed (FPKM $\geq 1$ ) gene number in whole body and antennal tissues of $\mathrm{H}$. phaleratus.

\begin{tabular}{llllll}
\hline Species & OBPs & CSPs & ORs & GRs & IRs/iGluRs \\
\hline H. phaleratus & $29(23)$ & $10(9)$ & $116(47)$ & $\mathbf{8 0 ( 3 0 )}$ & $35 / 13(26)$ \\
H. cichorii & 30 & 10 & 191 & 92 & $42 / 13$ \\
\hline
\end{tabular}

Table 2. The number antennal IRs of the six insects. Two major Hycleus-specific expansion of 'IR41a' and 'IR75' is evident. The number in bracket represents expressed (FPKM $\geq 1)$ gene number in whole body and antennal tissues of $\mathrm{H}$. phaleratus.

\begin{tabular}{lllllll}
\hline & H. phaleratus & H. cichorii & T. castaneum & D. ponderosae & C. lapponica & D. melanogaster \\
\hline IR25a & 1 & 1 & 1 & 1 & 1 & 1 \\
IR8a & 1 & 1 & 1 & 1 & 1 & 1 \\
IR75 & $10(7)$ & 16 & 3 & 4 & 4 & 4 \\
IR64a & 1 & 1 & 3 & 1 & 2 & 1 \\
IR40a & 1 & 1 & 1 & 1 & 2 & 1 \\
R21a & 1 & 1 & 1 & 1 & 1 & 1 \\
IR68a & 1 & 1 & 1 & 1 & 1 & 1 \\
IR41a & $11(5)$ & 15 & 2 & 1 & 1 & 1 \\
IR76b & 1 & 1 & 1 & 1 & 1 & 1 \\
IR93a & 1 & 1 & 1 & 2 & 2 & 13 \\
Total & 29 & 39 & $\mathbf{1 5}$ & $\mathbf{1 4}$ & $\mathbf{1 6}$ & \\
\hline
\end{tabular}

\section{Figures}




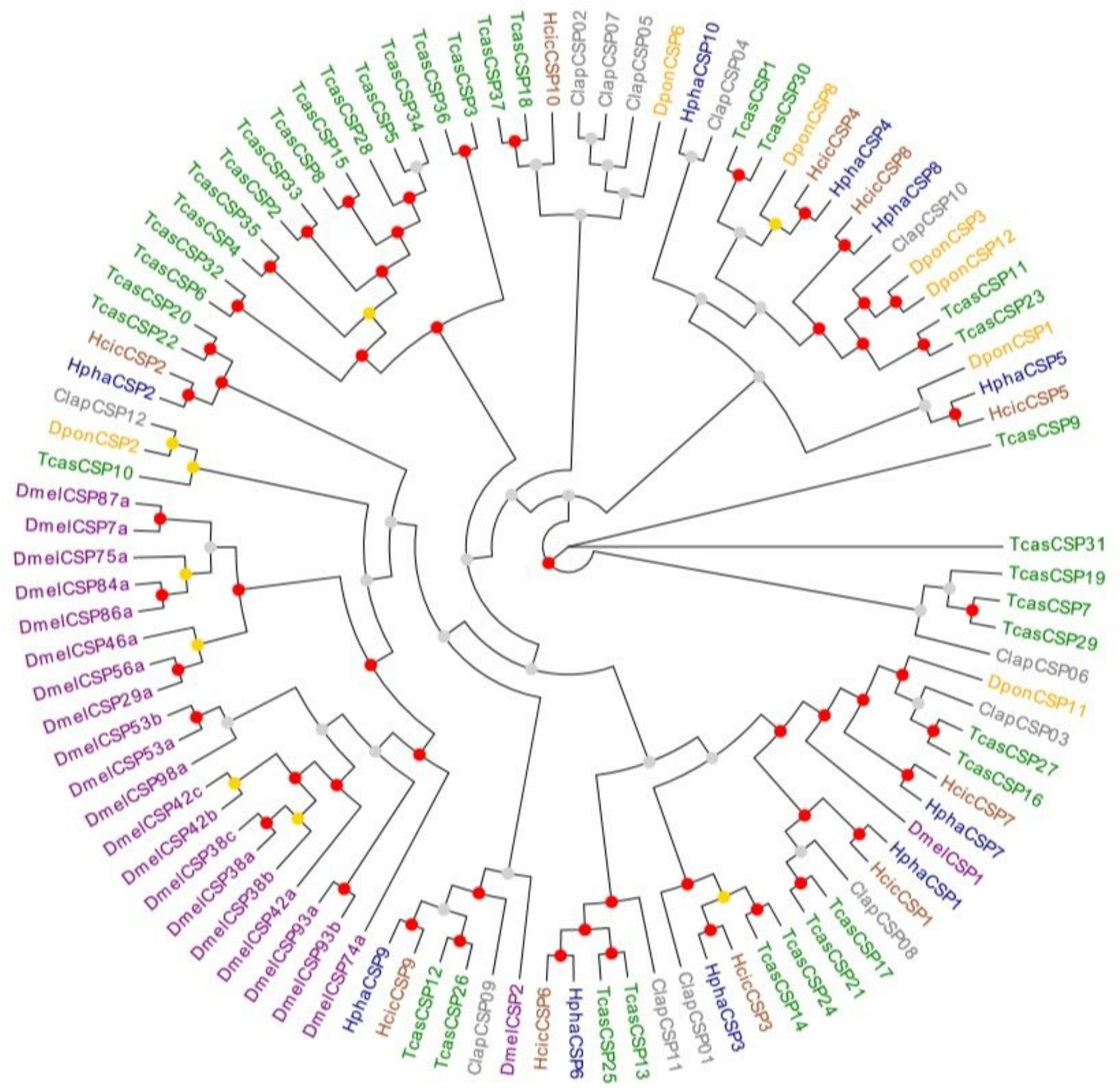

Figure 1

Figure 1. Maximum Likelihood Phylogeny based on the Protein Sequences of the Candidate Odorant Receptors (ORs). Red: H. cichorii (Hcic); blue: H. phaleratus (Hpha); grey: C. lapponica (Clap); green: T. castaneum (Tcas); yellow: D. ponderosae (Dpon). The colored dots refer to non-parametric bootstrap support (\%). Red: $₫ 70 \%$, yellow: $50-70 \%$ and grey: $₫ 50 \%$ support. 


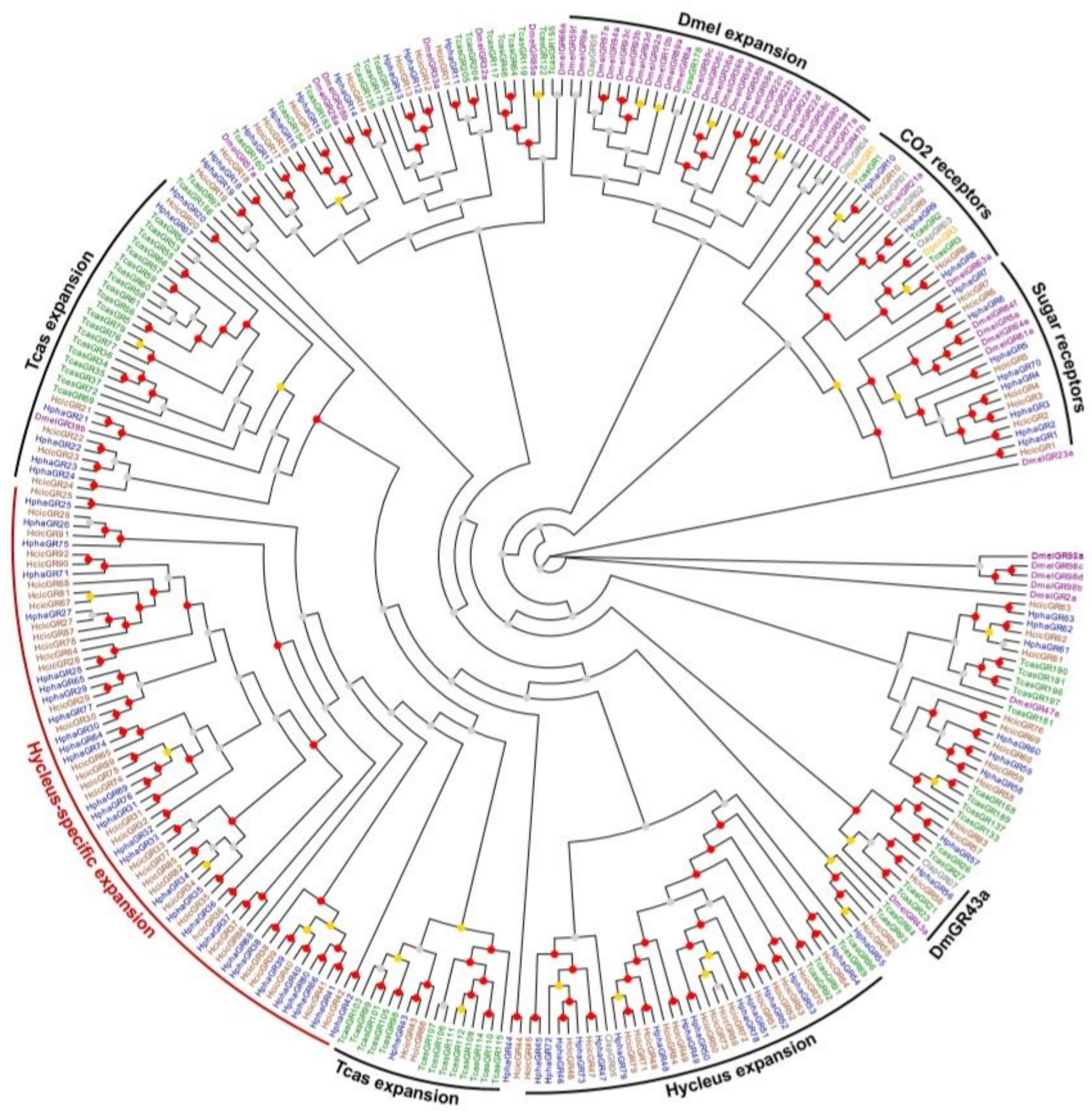

Figure 2

Fig 2. Maximum Likelihood Phylogeny based on the Protein Sequences of the Candidate GRs. Red: $\mathrm{H}$. cichorii (Hcic); blue: H. phaleratus (Hpha); grey: C. lapponica (Clap); green: T. castaneum (Tcas); yellow: D. ponderosae (Dpon) and purple: D. melanogaster (Dmel). One major Hycleus-specific expansion clade is evident (red). The colored dots refer to non-parametric bootstrap support (\%). Red: $₫ 70 \%$, yellow: $50-70 \%$ and grey: $₫ 50 \%$ support. 


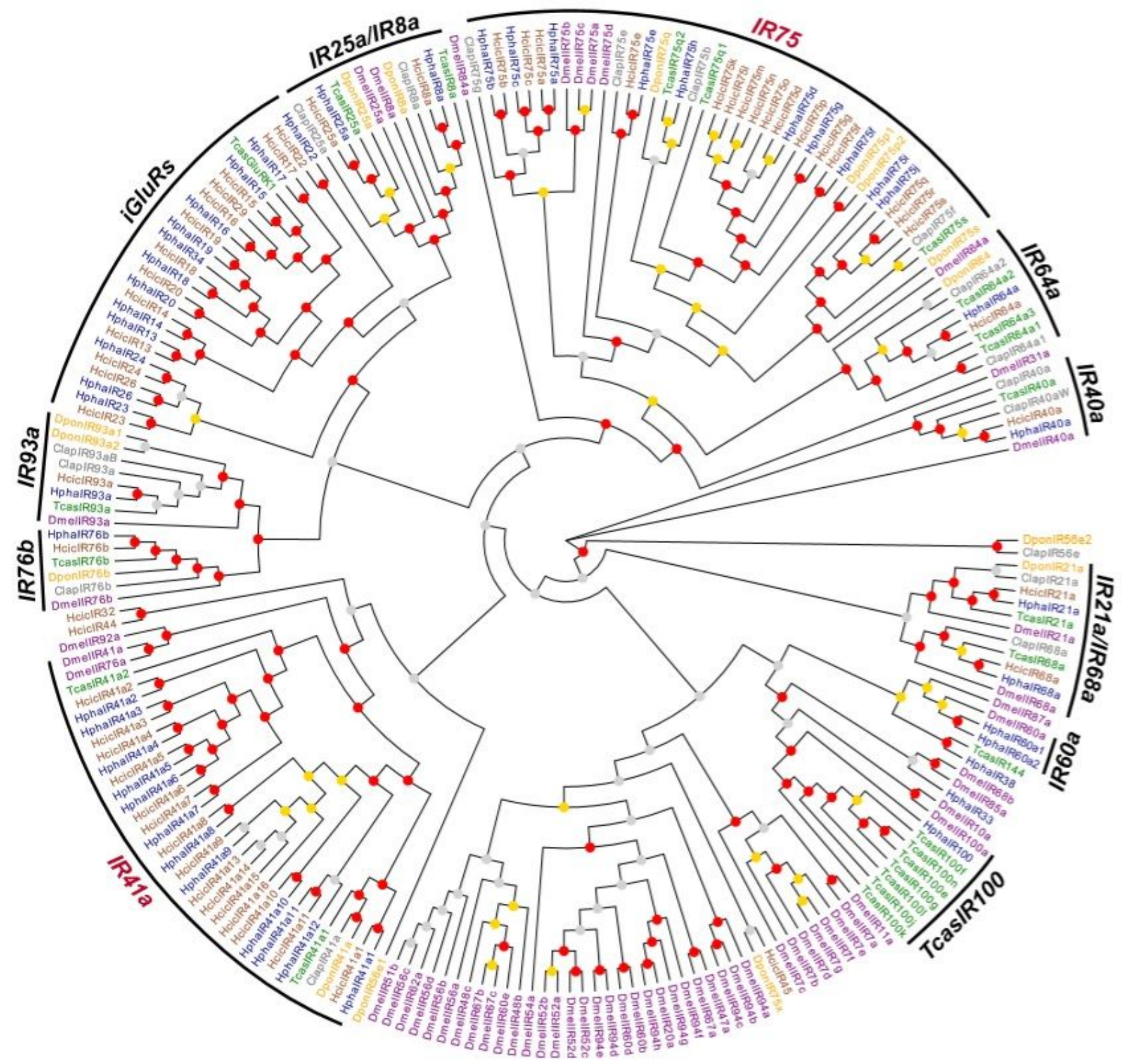

Figure 3

Fig 3. Maximum Likelihood Phylogeny based on the Protein Sequences of the Candidate IRs and iGluRs. Red: H. cichorii (Hcic); blue: H. phaleratus (Hpha); grey: C. lapponica (Clap); green: T. castaneum (Tcas); yellow: D. ponderosae (Dpon) and purple: D. melanogaster (Dmel). Two major Hycleus-specific expansion of 'IR41a' and 'IR75' clade is evident. The colored dots refer to non-parametric bootstrap support (\%). Red: $₫ 70 \%$, yellow: $50-70 \%$ and grey: $₫ 50 \%$ support.

\section{Supplementary Files}


This is a list of supplementary files associated with this preprint. Click to download.

- OR06282.pdf

- Additionalfiles3HycleuschemosensoryCDS.fa.gz

- Additionalfiles4Hycleuschemosensoryprotein.fa.gz

- OBP06212.pdf

- Additionalfiles2Kaksresultofallgenepairs.xls

- Additionalfiles1AllchemosensoryGeneFPKM.xls 